\title{
Estrategias de las mujeres del noreste de México para la sostenibilidad de la vida frente a la escasez del agua
}

\section{Northeastern Mexican Women's Life Sustainability Strategies Facing Water Scarcity}

\author{
Itzia María Cazares-Palacios* (D) https://orcid.org/0000-0002-0498-3820 \\ Karla Patricia Valdés-García* (i) https://orcid.org/0000-0002-1681-1954 \\ Alejandra de Arce ${ }^{* \star *}$ (D) http://orcid.org/0000-0001-5795-1403
}

\begin{abstract}
Resumen
Objetivo: conocer el punto de vista de las mujeres de Buñuelos, Coahuila, sobre la situación actual del agua en su localidad, los significados que otorgan al recurso y las estrategias que llevan a cabo para sostener la vida familiar y comunitaria frente a la escasez de éste. Metodología: siguiendo una metodología de tipo cualitativo, se realizaron entrevistas etnográficas y se conformaron grupos focales. Resultados: las formas de vida capitalistas limitan o restringen el acceso al agua a las mujeres, a la par que incrementan el trabajo que realizan para la reproducción social. Limitaciones: no se analizó ninguna otra dimensión estructural en torno a la calidad de vida en Buñuelos ni los aportes de otros actores clave de la localidad. Valor: la información descrita sirve como base para el diseño de políticas públicas orientadas a un desarrollo sostenible e incluyente. Conclusiones: los datos expuestos son importantes e imprescindibles debido a que provienen de las mujeres y desde el lugar que ocupan en la estructura social.
\end{abstract}

Palabras clave: sostenibilidad de la vida; estrategias; escasez de agua; género; noreste de México.

\begin{abstract}
Objective: to know Buñuelos, Coahuila, women's point of view regarding the current water situation in their locality, the meanings they give to this resource, and the strategies they carry out to sustain family and community life facing towards water scarcity. Methodology: following a qualitative methodology, ethnographic interviews and focus groups were conducted. Results: capitalist ways of life exclude women from the access to water and exacerbate their social reproduction-related work. Limitations: other structural dimensions around the quality of life in Buñuelos were not analyzed, neither the contributions of other female and male key actors in the locality. Value: the described information serves as the basis for public policies design aimed at sustainable and inclusive development. Conclusions: exposed data are important and essential due to they come from women and from the place they have in the social structure. However, the need for other studies that record the contributions of other female and male key actors in the locality is recognized, as well as a structural analysis of other dimensions of the quality of life in Buñuelos.

Keywords: sustainability of life; strategies; water scarcity; gender; Northeast Mexico.
\end{abstract}

Cómo citar: Cazares-Palacios, I. M.,Valdés-García, K. P., y Arce,A. de. (202I). Estrategias de las mujeres del noreste de México para la sostenibilidad de la vida frente a la escasez del agua. región y sociedad, 33, el 4l5. doi: I0.22/98/rys202I/33//4I5

*Autora para correspondencia. Universidad Autónoma del Estado de Hidalgo, Instituto de Ciencias de la Salud. Circuito ex-Hacienda La Concepción s. n., Carretera Pachuca Actopan, C. P. 42160. San Agustín Tlaxiaca, Hidalgo, México. Correo electrónico: itzia_cazares@uaeh.edu.mx

* Universidad Autónoma de Coahuila. Facultad de Psicología. Blvd. V. Carranza s. n., Col. República Oriente, C. P. 25280. Saltillo, Coahuila, México. Correo electrónico: karlavaldes@uadec.edu.mx

* Universidad Nacional de Quilmes. Centro de Estudios de la Argentina Rural. Consejo Nacional de Investigaciones Científicas y Técnicas. Roque Sáenz Peña Núm. 352, Bernal, Buenos Aires, Argentina. Correo electrónico: aledearce@gmail.com

Recibido: 5 de noviembre de 2020

Reevaluado: 18 de marzo de 2021

Aceptado: 23 de abril de 2021

Liberado: 1 de junio de 2021

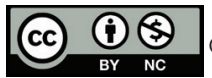

Esta obra está protegida bajo una Licencia

Creative Commons Atribución-No Comercial 4.0 Internacional. 


\section{Introducción}

El agua limpia y suficiente es un recurso esencial para la sostenibilidad de la vida y un derecho humano básico al cual todas las personas deberían tener acceso. No obstante, debido a factores estructurales, sociales, económicos, políticos y demográficos, desde las primeras civilizaciones hasta la actualidad (Gleick y Heberger, 2014), diversos contextos muestran lo contrario.

La escasez del agua es un problema grave a escala mundial que afecta las acciones para erradicar la pobreza y las desigualdades sociales, así como el logro del desarrollo sostenible. Entre los factores que provocan esta situación, están las causas meteorológicas, la contaminación, el deterioro de los ecosistemas, el aumento poblacional, la privatización y la mercantilización de los bienes naturales y la falsa representación de que el recurso es abundante (Esparza, 2014). Han recrudecido la problemática la construcción de grandes obras hidráulicas cuyo fin es extender la agricultura de riego y la producción de electricidad (Aboites, Birrichaga y Garay, 2010), la sobreexplotación de los acuíferos usados para la producción económica y el desarrollo social de la población (Aguirre, Tobón de Garza y Mendoza, 2018a y 2018b; Moreno, Marañón y López, 2010; Villarreal, 2013), así como la reducción de la responsabilidad del Estado de garantizar los derechos humanos (Ortega y Portillo, 2015).

Según el Informe Mundial de las Naciones Unidas sobre el Desarrollo de los Recursos Hídricos de 2018 (Organización de las Naciones Unidas para la Educación, la Ciencia y la Cultura [UNESCO], 2018), durante la década de 2010, 27\% de la población mundial vivía en áreas con mucha escasez de agua. En México, de acuerdo con ese documento, con información de la Secretaría de Medio Ambiente y Recursos Naturales (SEMARNAT) y de la Comisión Nacional del Agua (CONAGUA), en 2017, las regiones del centro, del norte y del noreste presentaban escasez de agua. Es relevante señalar que, según las fuentes citadas, estas zonas $^{1}$ concentran el mayor porcentaje de la población de México y generan la mayor aportación económica al producto interno bruto (PIB).

Así como la pobreza, la escasez de agua afecta de forma diferente a las mujeres y a los hombres debido a la división sexual y social del trabajo: las primeras son quienes realizan el trabajo doméstico y de cuidados, resultado de un orden social patriarcal.

A lo largo de la historia, las mujeres han sido "solucionadoras de problemas y preservadoras de la vida en lo cotidiano” (Segato, 2018, p. 16). Durante toda su vida se ocupan de garantizar la reproducción familiar en el espacio doméstico, "lugar que articula más y desde donde se puede acceder [o sea, tener acceso] a otros espacios productivos y relacionales" (Guzmán, 2005, p. 171) donde se crea plusvalía y riqueza económica a través de la economía del cuidado (Alberti, Zavala, Salcido y Real, 2014).

En relación con el agua, las campesinas garantizan este recurso para el sostenimiento de la vida familiar y comunitaria, pero debido a que lo obtienen por

1 Comprenden todos los estados del país, a excepción de Veracruz, Oaxaca, Guerrero, Chiapas, Tabasco, Quintana Roo, Campeche y Yucatán. 
lo general a través de medios precarios, invierten una cantidad considerable de tiempo y trabajo físico. Sin embargo, su participación en la formulación de políticas que determinan el acceso y el saneamiento del agua es mínima, o bien, cuando llegan a hacerlo, se las ubica en posiciones subalternas con escaso poder de acción y de decisión (García y Vázquez, 2017; Gutiérrez-Villalpando, Nazar-Beutelspacher, Zapata-Martelo, Contreras-Utrera y Salvatierra-Izaba, 2013; Ruiz, 2012; Sánchez y Fernández, 2018; Soares, 2007; Vázquez-Luna, Mortera, Rodríguez-Orozco, Martínez y Velázquez, 2013).

Desde hace casi tres décadas, en varias conferencias de las Naciones Unidas, ${ }^{2}$ se reconocieron las funciones que desempeñan las mujeres en el abastecimiento, la gestión y la protección del agua, y se indicó a los gobiernos y organismos socios ${ }^{3}$ que llevaran a cabo una serie de medidas para garantizar que las mujeres pobres tuvieran acceso al agua limpia, apta para el consumo, y que se las incluyera en las iniciativas de desarrollo.

Al respecto, Cleaver (1998) apunta varios aspectos: que las políticas derivadas de esos encuentros respondían a una situación macroeconómica mundial que favoreció la reasignación del papel del Estado debido a sus fallas para satisfacer las necesidades de los pobres en términos técnicos y ambientales sostenibles; que tales estrategias tienen un sesgo sectorial de valoración de los usos domésticos y productivos del agua; que esas políticas incorporan una versión que vincula la mayor participación de las mujeres pobres en la gestión del agua con la eficiencia y la productividad económica; que ninguna apunta a un cambio en las estructuras de género (Valdivieso, 2010); que pasan por alto los contextos estructurales e institucionales específicos que determinan el acceso y el control de los recursos (Gutiérrez-Villalpando et al., 2013; López y Rojas, 2017; Vázquez, Cárcamo y Buendía, 2007; Vázquez-Luna et al., 2013; Vázquez-García, Pérez-Olvera y Muñoz-Rodríguez, 2014; Vázquez-García y Sosa-Capistrán, 2017); y que omiten la diversidad de necesidades y preocupaciones de las mujeres con respecto a los medios para la subsistencia familiar.

Girón (2010) señala que las mujeres han sido la alternativa para las políticas económicas de estabilización neoliberales que han precarizado su inserción en el mercado laboral. También está el trabajo no remunerado que realizan para la sostenibilidad de la vida en reemplazo de un Estado que recorta los servicios públicos y sociales.

En el mismo tenor, a las mujeres se las convierte a menudo en destinatarias de las políticas públicas de desarrollo rural y urbano que las sitúan como responsables de su situación económica y social de partida y llegada; es decir, el Estado no discute las condiciones estructurales ni la pobreza, pero piensa a las mujeres como centro del hogar y agentes de desarrollo, cualidades que ve como parte de su "esencia femenina".

En la actualidad se enfrenta una crisis multidimensional mundial que, de acuerdo con Bartra (2013 y 2020), cuestiona la relación que tenemos con la

2 Conferencia Internacional de Dublín sobre Agua y Medio Ambiente en 1992; IV Conferencia Mundial sobre la Mujer de 1995 en Beijing; y en el periodo 2005-2015, la Declaración Internacional para la Acción El agua, Fuente de Vida.

3 Comisión Económica para América Latina y el Caribe (CEPAL) y Banco Mundial. 
naturaleza y que se expresa en la degradación medioambiental, en el estrés hídrico y en la pérdida de la biodiversidad. Ha empeorado la calidad de vida de sectores de la población vulnerados socialmente por razones estructurales. Se ha modificado la distribución entre Estado, empresas, familias y personas de las responsabilidades familiares sobre el bienestar de la población (Picchio, 2009). Se han recrudecido las desigualdades en las posiciones desde donde se reproduce la vida (Carosio, 2010). Todo lo anterior demuestra la incapacidad del modelo económico capitalista para dar respuesta a las necesidades y a las condiciones vitales de las personas (Carrasco, 2016).

La sostenibilidad de la vida, cuyo interés central es el cuidado de ésta y la reproducción humana como proceso social, es un enfoque que cobra relevancia en este trabajo.

Desde una perspectiva económica feminista que advierte los sesgos androcéntricos y capitalistas que caracterizan a la economía clásica y neoclásica, y cuya propuesta política es la sostenibilidad de las condiciones y los procesos de reproducción de la vida humana y no humana, Carrasco (2011, 2014 y 2016) y Picchio (2009) especifican que la cuestión de la subsistencia en sus dimensiones materiales, sociales y afectivas es básica para la definición de cualquier sistema social.

Carrasco (2016) señala tres ámbitos de los que depende la vida de las personas y que constituyen la base del sistema económico y social: la naturaleza, el espacio de lo doméstico y los cuidados, y el ámbito de la producción y la transformación en las esferas privada y pública. Sin embargo, la autora indica que las sociedades patriarcales capitalistas han privilegiado el beneficio económico sobre las condiciones de sostenibilidad de la vida, y han ocultado las relaciones de explotación y depredación que establece la producción capitalista con el ámbito del cuidado y la naturaleza, respectivamente. La autora advierte que la naturaleza y las personas no son un objetivo social prioritario, sino que están al servicio de la producción.

En el caso de las personas, de acuerdo con Picchio (2009), eso no significa que se adapten de forma mecánica o autómata a los procesos de producción, sino que, motivadas por sus necesidades, deseos, aspiraciones, percepciones de vulnerabilidad, vínculos y relaciones, reaccionan de manera independiente, es decir, con agencia.

De ahí que, frente a las múltiples crisis de supervivencia (Carosio, 2010) que enfrenta la humanidad como resultado del modelo económico capitalista, patriarcal y neoliberal, es necesario que los análisis no sólo se concentren en la producción de los medios para la sostenibilidad de la vida, sino también en las fuerzas subjetivas y en las condiciones de vida que movilizan y subyacen a los procesos de vida de las personas. Según Picchio (2009), se trata de "una ética consustancial con los comportamientos individuales, como algo no eliminable; como sentido de la producción y distribución de los medios de vida" (p. 35).

Como se mencionó, a lo largo de la historia las mujeres han realizado los trabajos de los que depende de manera directa la vida de las personas, por lo que, en términos epistémicos, el enfoque de la sostenibilidad de la vida encuentra 
lugar en la propuesta de los conocimientos situados (Haraway, 1995), planteada a partir de la teoría feminista del punto de vista (Harding, 2010).

Dicha teoría afirma que la posición subalterna de las personas en la estructura social promueve la producción de conocimiento alterno al enfoque androcéntrico y capitalista (Harding, 2010). De esta forma, se reconoce el lugar privilegiado de las mujeres en la construcción de conocimientos situados, con lo cual es posible revertir los sesgos androcéntricos de los paradigmas científicos dominantes y producir conocimiento parcial pero objetivo con y para las mujeres, con el fin de "prometer versiones más transformadoras, más adecuadas, sustentadas y objetivas del mundo" (Haraway, 1995, p. 328).

La creación de conocimiento con y para las mujeres tiene una implicación política poderosa, porque permite visibilizar sus necesidades, intereses, motivaciones, los significados que imprimen a sus acciones y sus márgenes para actuar, de tal forma que se puedan tomar medidas que contribuyan a la construcción de realidades más equitativas y justas.

Con base en lo expuesto, los objetivos de esta investigación son conocer las percepciones de las mujeres de Buñuelos sobre la situación actual del agua en su localidad, los significados que le otorgan y las estrategias individuales y colectivas para enfrentar el problema de la escasez de un recurso necesario para sostener la vida familiar y comunitaria.

\section{Metodología}

Se siguió una metodología cualitativa, basada en un enfoque crítico e interpretativo (Flick, 2004), con un alcance exploratorio que permitió describir los significados, las percepciones, los recursos, las estrategias y la capacidad de acción de las mujeres de la localidad de Buñuelos para enfrentar las dificultades cotidianas y sostener la vida familiar y comunitaria. También se investigaron los obstáculos de género que enfrentan para participar de forma pública en la toma de decisiones sobre la mejora de las condiciones de abastecimiento y saneamiento de agua en la localidad.

Para lograrlo, se realizaron observaciones participantes (Martínez, 2007), registradas en diarios de campo, entrevistas semiestructuradas de tipo etnográfico (Flick, 2004) y se crearon grupos focales (Martínez, 2004). En conjunto, las técnicas descritas constituyen una triangulación de datos cuyo propósito es encontrar consistencias en los resultados y fortalecer la validez interna de la investigación (Okuda y Gómez-Restrepo, 2005).

Las entrevistas semiestructuradas y los comentarios de los grupos focales se transcribieron, codificaron y categorizaron (Kvale, 2007) en los siguientes focos temáticos: a) percepciones de las mujeres sobre la situación actual del agua; b) significados que otorgan a ésta; c) estrategias individuales y colectivas para enfrentar su escasez; y d) prejuicios de género que limitan la participación y la toma de decisiones de las mujeres en la esfera pública. Después la información se trató mediante un análisis cualitativo (Cáceres, 2008). 
Dado el propósito de este estudio, la meta es una generalización teórico-analítica, por lo que el muestreo es no probabilístico selectivo o intencionado ( $\mathrm{Pa}$ tton, 1990). La estrategia para identificar a las participantes fue mediante la organización de reuniones con la comunidad y con cada una de ellas de manera directa. Una vez identificadas las posibles participantes, se les planteó el propósito del estudio, y a quienes aceptaron se les solicitó su consentimiento oral para audiograbar las entrevistas individuales y las conversaciones de los grupos focales.

Se realizaron 15 entrevistas individuales en los domicilios de las participantes y se conjuntaron dos grupos focales, cada uno integrado por diez participantes, mismos que se llevaron a cabo en la Escuela Primaria Comunitaria de la localidad.

El trabajo de campo se realizó entre septiembre y diciembre de 2019. ${ }^{4}$ Participaron 35 mujeres originarias de Buñuelos, con edades de entre 20 y 56 años -con una media de edad de 38 años-. A excepción de una participante, todas tienen entre 1 y 5 hijos cuyas edades oscilan entre 6 y 32 años. La mayoría de las mujeres están casadas y sólo 2 se encontraban viviendo en unión libre. Los hogares donde habitan las participantes son nucleares y ampliados.

En relación con la organización familiar, todas se dedican al trabajo doméstico y a las labores de cuidado. No obstante, algunas reportaron emplearse de forma temporal en la pavimentación de carreteras o bien haciendo comida para trabajadores de la construcción. La mayoría de sus parejas e hijos varones mayores de edad trabajan en el Parque Industrial Derramadero y los otros, como albañiles y en labores de cortadillo. ${ }^{5}$

\section{Contexto de trabajo}

De acuerdo con el Censo de Población y Vivienda 2020, de 3146771 habitantes de Coahuila, 92\% vive en contextos urbanos y $8 \%$ en territorio rural (Instituto Nacional de Estadística y Geografía [INEGI], 2020). ${ }^{6}$

Coahuila se encuentra en zonas áridas y semiáridas. Sus suelos están relacionados con los matorrales desérticos micrófilo y rosetófilo, y en menor medida con la vegetación de desiertos arenosos. Presenta un clima muy seco (46\%), seco y semiseco (49\%) y templado subhúmedo (5\%). Tiene un promedio de 350

4 Durante febrero y mayo de 2019, profesoras y estudiantes de diversas facultades del área de Ciencias Sociales de una Universidad Pública de México, acudimos a Buñuelos en el marco de unas jornadas rurales, con el objetivo de realizar encuestas y entrevistas sobre el bienestar y calidad de vida de la población. Durante el trabajo de campo se realizaron reuniones con personas de la comunidad a fin de conocer las necesidades que enfrentaban respecto a los servicios básicos, así como en relación con problemáticas psicosociales. En su mayoría quienes asistieron fueron mujeres jefas de familia que destacaron el tema de escasez de agua. Esto dio lugar al objetivo de la presente investigación.

5 Fibra cuyo uso puede ser artesanal para la elaboración de canastas o industrial y que junto con el sorgo escobero es utilizado para la elaboración de escobas y cepillos.

6 Cabe señalar que los estados limítrofes del norte del país concentran un porcentaje inferior a $25 \%$ de la población rural, en contraste con aquellos que están en la franja sur y centro, que aglutinan el mayor porcentaje de este sector (Consejo Nacional de Población [CONAPO], 2012). 
milímetros $(\mathrm{mm})$ de precipitaciones pluviales al año y en algunas regiones se tienen temperaturas en verano por arriba de los $40^{\circ}$ Celsius y en invierno por debajo de cero. Coahuila contaba en 2016 con una capacidad de agua renovable de 3185 hectómetros cúbicos por año ( $\mathrm{hm}^{3} /$ año) y de $1063 \mathrm{hm}^{3}$ por habitante al año ( $\left.\mathrm{hm}^{3} / \mathrm{hab} / \mathrm{año}\right)$. Si se compara con los estados ubicados al sur del país, como Chiapas, donde predominan selvas húmedas en las cuales se presentan $113903 \mathrm{hm}^{3} /$ año y $21419 \mathrm{hm}^{3} / \mathrm{hab} /$ año (Gobierno del Estado de Coahuila de Zaragoza, 2018; SEMARNAT y CONAGUA, 2017), puede verse la diferencia.

En términos hidrológicos, Coahuila es parte de cuatro zonas: Río Bravo-Conchos, Mapimí, Nazas-Aguanaval y El Salado. Posee una red grande de arroyos, en mayor medida intermitentes y en algunos casos perennes, que conducen el agua a través de las cuencas a lo largo del estado y que favorecen la recarga de los acuíferos. Es destacable indicar que, de acuerdo con González (2017), en la actualidad, los acuíferos del estado enfrentan una problemática grave por su sobreexplotación agrícola y de generación de electricidad pública e industrial. Los más afectados son el Principal-Región Lagunera, Monclova y Saltillo-Ramos Arizpe.

Coahuila se divide en cinco regiones geográficas: fronteriza-norte, carbonífera, centro-desierto, Laguna y sureste. En esta última se sitúa el presente trabajo y comprende los municipios de Arteaga, General Cepeda, Saltillo, Ramos Arizpe y Parras.

Figura 1. Localización geográfica de Buñuelos, Coahuila
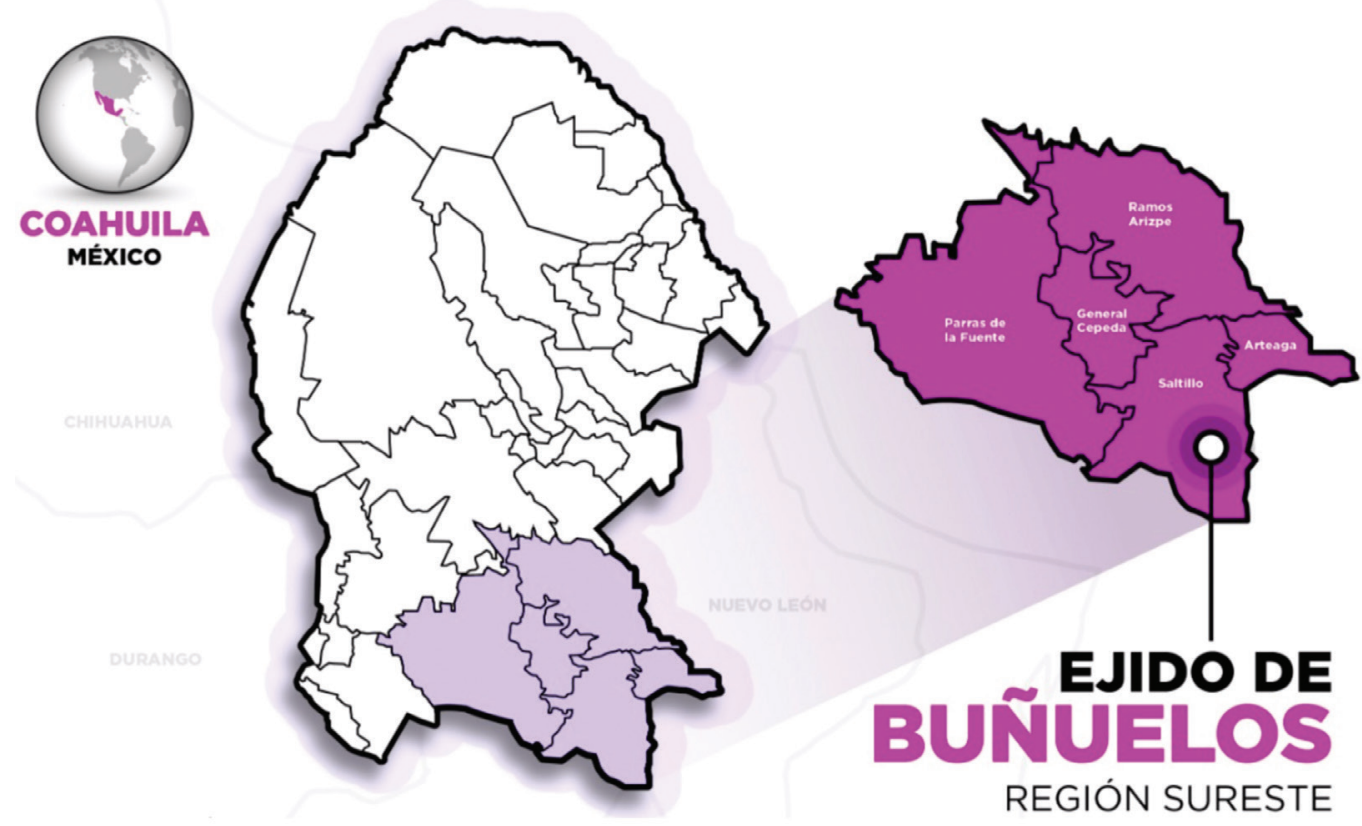

Fuente: elaboración propia. 
En términos económicos, la región sureste del estado está dinamizada por la industria manufacturera automotriz. Las actividades económicas secundarias tributan $53.6 \%$ al PIB del estado. Le siguen las terciarias con $43.9 \%$ y las primarias con $2.4 \%$. Dos municipios de esta región, Saltillo y Ramos Arizpe, aportan juntos 50.7\% del valor de la producción bruta total (INEGI, 2016).

Buñuelos, lugar donde se realizó el estudio, es una localidad rural que pertenece al municipio de Saltillo. Se encuentra al sureste de la capital de Coahuila y colinda al norte con el ejido Agua Nueva, al sur con el ejido Gómez Farías, al este con el ejido Las Colonias y al oeste con el ejido Tinajuelas.

En 2010, Buñuelos contaba con 194 habitantes, 108 hombres y 86 mujeres, distribuidos en 50 viviendas, y mostraba un grado de marginación alto. De quienes tienen entre 15 años o más, $21.50 \%$ presentaba analfabetismo, $44.70 \%$ tenía primaria incompleta y 9.52\% de la población de entre 6 a 14 años no asistía a la escuela. No disponía de drenaje $76 \%$ de las viviendas, $16 \%$ no contaba con inodoro, $2 \%$ no tenía energía eléctrica ni agua entubada y $36.08 \%$ no tenía derechohabiencia a los servicios de salud (Secretaría de Desarrollo Social [SEDESOL], 2013).

De acuerdo con el trabajo de campo realizado, se observó que la red de abastecimiento de agua es deficiente, razón por la que las familias acuden a los ejidos cercanos para conseguirla.

Son pocas las familias que tienen huertos de traspatio, cosecha de nopal o crianza de cerdo y gallinas destinada al autoconsumo. La mayor parte de la población activa en términos económicos se emplea en el sector formal industrial como operarios en el Parque Industrial Derramadero, que se encuentra más o menos a 37 kilómetros de distancia de Buñuelos. En menor medida, como se mencionó, algunos hombres de la localidad trabajan como albañiles y en labores de cortadillo.

Figura 2. Secado de la hoja de cortadillo en la localidad de Buñuelos

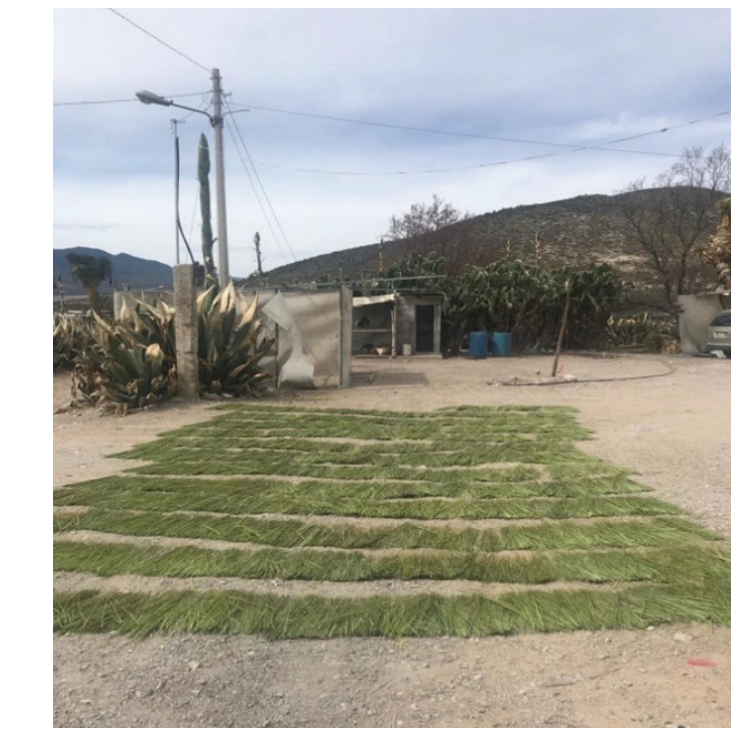

Fuente: fotografía de Itzia María Cazares Palacios, 2019. 
En términos hidrológicos, en el momento de realizar el trabajo de campo sólo fue visible un estanque cuya agua se utiliza para algunas actividades domésticas y para el ganado. Sobre el abastecimiento de agua, la comunidad de Buñuelos, a través de su Comité de Agua, la trae de los pozos ubicados en los ejidos de San Felipe, Carneros y Notillas. Según información proporcionada por las participantes de este estudio, el pozo que suministraba el agua está contaminado con residuos de rocas fracturadas y sales, razón por la que acuden a los ejidos mencionados.

Figura 3. Estanque visible de Buñuelos, Coahuila

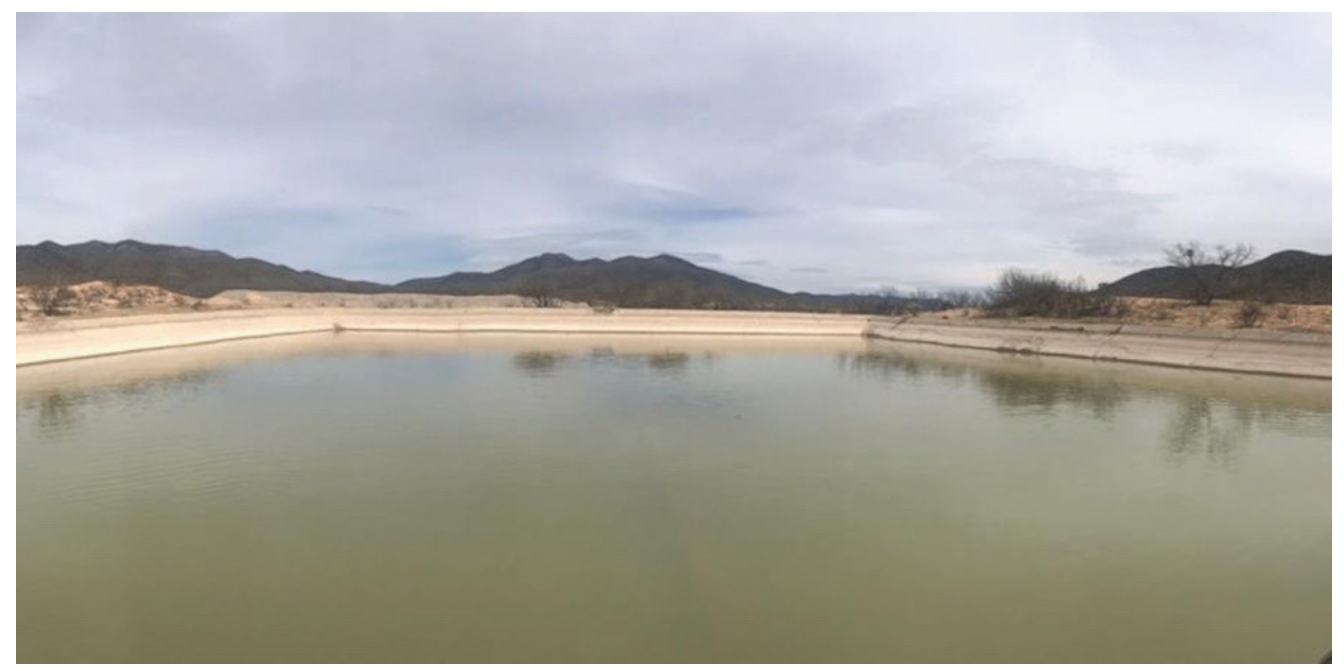

Fuente: fotografía de Itzia María Cazares Palacios, 2019.

Buñuelos forma parte de los municipios aledaños a la zona industrial Alianza Derramadero, la cual alberga cinco parques industriales en el giro manufacturero automotriz. En la actualidad, esta zona se integra al proyecto Ciudad Modelo Derramadero, que planea la creación de viviendas y servicios básicos para la población trabajadora de esas empresas. Es importante señalar que, según el "Informe Derramadero" de la Fundación de Villa de Patos, Asociación Civil, A. C., y el Instituto Mexicano para el Desarrollo Comunitario (2018), la población rural asentada en esa zona, así como la trabajadora que se concentrará ahí, enfrentará problemas graves para la captación, la distribución y el suministro de agua, debido a que las cinco zonas de captación y recarga de los acuíferos que abastecen a la zona metropolitana de Saltillo, ${ }^{7}$ registran niveles de abatimiento significativos. Esta situación se agravará en años próximos, considerando el aumento poblacional.

7 Se trata de los acuíferos de Agua Nueva, Carneros, Loma Alta, San Lorenzo Terneras y Zapalinamé. 
Villarreal (2013) respalda lo descrito. Indica que el valle de Saltillo, al no contar con presas, lagos, ríos o arroyos para soportar el crecimiento poblacional asociado con el desarrollo del complejo industrial Derramadero, la sobreexplotación actual de los acuíferos, así como por la baja eficiencia en sus sistemas de distribución, enfrentará consecuencias perniciosas para el consumo doméstico y la producción, tal como indican Aguirre, Tobón de Garza y Mendoza (2018a y 2018b).

\section{Análisis de resultados}

Percepción de la situación actual del agua en Buñuelos

El tema inicial explorado tanto en las entrevistas individuales como en los grupos focales fue la situación del agua en la localidad. Salvo una participante, las demás son originarias de Buñuelos y han radicado ahí toda su vida. Con base en sus experiencias cotidianas, las mujeres realizan un análisis de la situación del agua a través del tiempo.

Pues mira, cuando nosotras estábamos muchachas, en la casa acarreábamos agua - para lavar, para trapear, para el quehacer- del estanque que está aquí abajo; y para tomar, de otro que le decimos El Talú [talud] [...]. Y de ahí, acarreaba uno agua para tomar, pero, pues nada más la colábamos y ya era la que tomábamos aquí [...]. Sí ha mejorado porque ahora, pues, ya la tenemos en tubería. Le digo, el problema aquí es que se tardan mucho para echar el agua, de hecho, pos ya venden agua purificada. Ya la que no quiere tomar de la llave, porque de repente está sucia la pila o se le meten animalitos o así, ya la compra uno en la tienda. (Mujer de 42 años)

Los abuelos tomaban de los estanques y del Talud [...]. Era más limpia, sobre todo El Talud. De ahí tomaban, pero, pues antes tenía malla, o sea, estaba más limpio, y ahora ya no [...]. Antes sí nos dábamos abasto, pero ahorita ya son muchas familias [...]. Creo que actualmente existen como unas setenta familias y antes eran unas poquitas, como treinta [...]. (Mujer de 53 años)

Como se puede apreciar, en la actualidad el agua de Buñuelos es menos limpia y segura para el consumo, lo cual genera un costo económico para quienes tienen la posibilidad de comprar agua de garrafón purificada. Para quienes no tienen esa opción, es un riesgo para su salud consumir el agua no purificada. El incremento de la población entre 2010 y 2019 ha provocado una menor disponibilidad de ésta para el consumo de las familias. Así mismo, las participantes identifican efectos negativos para el sostenimiento de la vida no humana provocados por la industria. 
Como que antes llovía más, entonces, en ese tiempo ellos, los que tenían, sí tenían mucha agua para recolección y acarrear [...]. Mi esposo dice que Derramadero está afectando el consumo del agua, porque es la misma agua [...]. Mi esposo todo el tiempo que trabajó en Derramadero y también en la cosecha de la papa, ha notado esos efectos [...]. Nosotros vivimos en la manzana en Agua Nueva. Se acabó todo. Se secaron árboles. (Mujer de 44 años)

Cabe destacar que, de acuerdo con Villarreal (2013), en la década de 1960, Buñuelos presentaba un índice de eficacia de gestión del agua de 0.85 , mientras que en 2012 era de 0.5, depreciación que, según el autor, es un efecto de la acción antropogénica sobre el agua del subsuelo, con consecuencias adversas sobre la salud de sus habitantes, cuya producción agrícola y calidad de vida se modificaron.

La evaluación actual que las mujeres realizan de la disponibilidad del agua está marcada por sus propios tiempos de vida familiar. La escasez se vincula con rememoraciones del pasado, pero también con las variaciones en las temporadas de lluvia, como resultado del cambio climático, que derivan en reducciones de precipitaciones pluviales.

Por un lado, en el caso de las abuelas y los abuelos, se vio afectada la libertad de disponer de agua de fuentes naturales debido al cambio climático, que produce escasez de lluvia. También mencionan la contaminación del agua y la posibilidad, para algunos, de tener acceso al agua embotellada. Esto último muestra cómo la imposibilidad de disponer del agua de manera natural precariza la reproducción social de los miembros de la localidad en la medida en que se mercantiliza el acceso. En consecuencia, recrudece las desigualdades económicas en Buñuelos e incrementa la carga de trabajo en las mujeres en la medida en que la disponibilidad del agua ha disminuido.

El aumento poblacional y su impacto, así como el establecimiento de la industria y el elevado consumo, son condiciones que afectan el acceso al agua. También se encontró que las mujeres jóvenes y las mayores están conscientes del esfuerzo que conlleva tenerla para el consumo familiar, tarea que recae en ellas.

Significado del agua

El significado que dan las mujeres al agua es fundamental para comprender sus acciones y sus funciones sociales, que se traducen en trabajo doméstico y en los cuidados para lograr el sostenimiento de la vida familiar. Comentaron que el significado es:

Vida, porque sin agua no somos nada; o sea, no tomamos agua y nos deshidratamos, nos enfermamos y ¿con qué cocinamos? Tampoco comemos. Para bañarnos, como quiera, podemos buscar o juntamos, ir ahí al talú (talud). Pero la necesidad es para tomar y para cocinar [...]. Es vida para las familias. (Mujer de 42 años) 
Pues todo. Sin agua no hacemos nada [...]. El agua es muy necesaria para todo, hasta yo creo más que el dinero, porque sí, porque el agua es para todo y para todos [...]. Si una no tiene agua, ¿con qué cocina, lava? ¿Con qué se baña? ¿Cómo se lava los dientes? ¿Con qué hacen una comida? (Mujer de 44 años)

El agua es como la vida [...]. Si no tienes agua, no tienes nada [...]. Nos ha pasado el caso, cuando no tenemos de la llave, que es tiempo de sequía, que no hay en los tanques ni nada. Los señores también batallan mucho para acarrear agua para sus animales, porque tienen que buscar donde haya agua para darles a los animales, porque si andan en el monte, pues también se mueren porque ellos, aunque busquen agua y si están secos los estanques, pues se mueren en el monte los animalitos. Sí, pues el agua es todo. (Mujer de 42 años)

El agua es un recurso imprescindible para la sostenibilidad de la vida humana y no humana en la cotidianidad de las participantes. Su consumo permite que sus cuerpos funcionen de forma saludable y realicen diversas actividades domésticas y de cuidado, como cocinar, bañar, lavar. También permite la reproducción de la vida de los animales que forman parte de su subsistencia. El agua es más valiosa en sus vidas que el dinero, porque reconocen que es un recurso natural, base de su sistema y funcionamiento social. En este sentido, considerando que este recurso es fundamental para el bienestar de todos, su desabasto dinamiza acciones colectivas para enfrentarlo y resolverlo.

Estrategias individuales y colectivas para enfrentar la escasez de agua

¿Qué hacen las mujeres para sostener la vida familiar y comunitaria frente a la escasez de agua? ¿En qué condiciones y cuáles son las implicaciones de estas actividades de trabajo para sí mismas y en relación con el otro? Son preguntas clave para evidenciar lo que subyace a los procesos de reproducción social y de mantenimiento de la vida.

Las participantes coinciden en el tipo de estrategias organizativas y de administración del agua para llevar a cabo el trabajo doméstico y de cuidados.

Uno [tinaco] lo lleno para el baño, que es el que está arriba del baño y lleno el otro que esta acá atrás, que es el que uso para lavar y así. Y el otro que uso para el agua de la cocina, es para tomar, para los trastes. (Mujer de 42 años)

De la llovediza agarro para el baño, para bañarnos, para trapear, para lavar trastes, lavar [...]. Cuando vamos al talú (talud) o a Carneros, vamos juntos mi esposo y mis hijos [...] y pos de a tinita. Una tinita para lavar los trastes, una tinita para hacer tortillas, porque nosotros aquí todos los días hacemos tortillas, mañana, medio día y noche. Se hace tortilla cuando hay maíz de molino y si no, pues de Maseca, pero pos todo el día usamos el agua. (Mujer de 49 años) 
En diciembre, que no había, llovía y yo llenaba un tonel aquí y lo tapaba. Le ponía un hule y de ahí para tomar. (Mujer de 44 años)

Algunas mujeres recolectan el agua de lluvia para el consumo físico; otras la utilizan para las tareas domésticas. La diferencia en el uso se relaciona con las posibilidades materiales y económicas de cada familia. Como se mencionó, el pozo de Buñuelos está contaminado, razón por la que algunas familias que tienen los medios propios para trasladarse, acuden a otros lugares, una o dos veces a la semana, para obtener el agua de los pozos que están ahí. Hay algunas familias que tienen ingresos suficientes para comprar agua purificada de garrafones para el autoconsumo. Cabe señalar que, según lo reportado por las participantes, los organismos estatales y municipales no han implementado mecanismos educativos para asegurar la salubridad en el manejo del agua.

En relación con la organización familiar para la obtención del agua, son las mujeres quienes se ocupan de poner los tinacos para recolectar el agua de la lluvia, mientras que los esposos o parejas e hijos participan cuando es necesario trasladarse a los pozos, más o menos a cuarenta minutos de distancia, o al abrevadero (talud).

Con respecto a las estrategias colectivas, las mujeres están al pendiente y avisan a las otras y al Comité del Agua que van a abastecer el estanque. Ellas llenan los tinacos y sus parejas e hijos, por lo general los varones, se encargan de trasladarlos a sus casas. Estas actividades las realizan de una a dos veces a la semana.

Nos organizamos entre varias, así como nos juntamos en las juntas de la escuela, así nos juntamos. Nos avisamos una con otra: "Oye, va a haber junta" y ya corren la voz o si es posible, que dicen "No, pues, hazme el favor tú de avisarle" [cuando van a echar el agua]. Pues ya andamos casa por casa y ya nos juntamos todas a tal hora, y ya vamos a llenar los tinacos y nos acompañan nuestros esposos, si están, o con apoyo de nuestros hijos. (Mujer de 49 años)

Cuando llega a estar contaminada el agua, vamos y lavamos la pila, la pila de donde viene el agua. Nos organizamos todos, o sea, todo el rancho, todas las mujeres, los hombres. Nos organizamos. (Mujer, 49 años)

Por otra parte, las mujeres toman la iniciativa de organizarse para limpiar el estanque que distribuye el agua y arreglar las averías en las tuberías cuando no es posible que el comisariado lo haga.

Cuando se desbarata el pozo, a veces que anda ocupado el comisariado, dice una: "Júntense dos o tres mujeres y van a hablar para preguntar del fierro”, y así. (Mujer de 53 años)

Es importante precisar que, cuando hay alguna avería en la bomba o pozo, si es el caso, las mujeres se reúnen y van a buscar al comisariado a su casa para que la componga. Sin embargo, cuando no lo encuentran o reciben una negati- 
va, con el conocimiento y las estrategias de que disponen, intentan arreglarla, aunque no siempre lo logran.

Participación de las mujeres en la toma de decisiones públicas para el abastecimiento y el saneamiento de agua

A pesar de las distintas acciones que llevan a cabo las mujeres para enfrentar la escasez del agua y sostener la vida familiar y comunitaria, se observa una estructura patriarcal y prejuicios de género en relación con las capacidades de las mujeres. Esta situación dificulta que ellas formen parte del Comité del Agua para mejorar los servicios de abastecimiento y saneamiento de agua.

Es el comisariado, el delegado y el juez y el secretario [...]. Ellos, o sea los hombres, hacen las juntas entre ellos, y entre ellos mismos deciden el Comité [...]. En las juntas, van más mujeres que hombres [...]. Las mujeres somos las que batallamos con el agua. Ellos, como quiera, ellos comen, ellos duermen, ellos se van a trabajar y uno es la que batalla del agua [...]. Sí estaría bien que una mujer estuviera al frente del Comité del Agua [...]. Si yo fuera encargada, yo voy y la prendo [la bomba de agua], echo el agua a la gente y ya me regreso a mi casa. Sigo con mis actividades, echo el agua, voy y le apago a la bomba. Le echo al agua, que agarre el agua la gente, me regreso y sigo con mis cosas [...] porque las que manejamos el agua somos las que debemos de movernos. (Mujer, 49 años)

Aquí las señoras comentan que las juntas las deben de llevar las mujeres, y los señores dicen "No. No". Y sí es cierto, porque nos ha pasado que las señoras discutimos mucho, ni arreglamos, y a veces, puro chisme [...]. Aquí el control siempre lo han llevado unos señores. (Mujer, 47 años)

Los hombres llevan más el control, y bueno, es como cuando hacemos un aporte y entramos en puros conflictos, no nos ponemos de acuerdo. $Y$ luego aquí se dirige por ejidatarios, ellos ya tienen el dominio [acuerdos establecidos] [...] hasta que llegan los domingos, y así ya se llevan el control, y las mujeres, pues no. Las mujeres somos más problemáticas, más chismosas. (Mujer, 46 años)

Se observa el reconocimiento que hacen las mujeres a la importancia de su participación en el Comité del Agua, porque ellas realizan las funciones domésticas y comunitarias para la reproducción social. Estas acciones, además, posibilitan el ejercicio de un poder en ellas, entendido como un potencial para, es decir, un poder que suma en lo colectivo. No obstante, es notable el carácter patriarcal de este comité, que limita la representación y participación de las mujeres en él.

También pueden leerse en los comentarios las dificultades en la comunicación, negociación y solución de conflictos entre las mujeres, así como el chisme, que entorpecen su organización colectiva y construyen su autopercepción 
como incapaces para dirigir este espacio de poder. Las implicaciones de estas formas de violencia simbólica, en las cuales las mujeres se apropian y reproducen entre ellas un discurso patriarcal que desacredita sus capacidades y su voz, es un mecanismo que sostiene y calca una estructura patriarcal que las excluye de participar en asuntos públicos.

\section{Discusión y conclusiones}

En su alcance exploratorio, este trabajo constituye una aproximación a la realidad de vida de las mujeres de Buñuelos, en el noreste mexicano. En especial, reveló y permitió el registro de los significados que las mujeres dan al agua para el sostenimiento de la vida familiar y de sus experiencias y percepciones en torno a la situación actual de este recurso en su localidad. Así mismo evidencia la capacidad de agencia que tienen, es decir, su iniciativa, sus acciones y las estrategias que llevan a cabo en la vida diaria para enfrentar la escasez del recurso, entre otras tareas de cuidado que realizan.

La teoría del punto de vista feminista de Harding (2010) y la propuesta de los conocimientos situados de Haraway (1995) fueron el punto de partida para advertir que, de cara a la crisis civilizatoria que enfrenta la humanidad, no se pueden entender las sociedades y la historia desde el punto de vista de un sujeto universal y atemporal. Gracias a ambas propuestas se puede distinguir el límite del proyecto societario de la modernidad, así como la necesidad de un nuevo sentido civilizatorio y la refundación del modelo económico prevaleciente (Federici, 2010, 2018 y 2020).

Con base en lo anterior, en este apartado se discuten los resultados de este estudio a la luz de las principales críticas feministas al capitalismo patriarcal, liberal y neoliberal desde el enfoque de la sostenibilidad de la vida humana y no humana.

Desde la década de 1980, como resultado de las políticas neoliberales favorables al capital en América Latina, se advirtió un proceso violento de reproducción del capital que va de la mano de un embate de nuevos cercamientos (Federici, 2010 y 2020), que precarizan y minan toda posibilidad de reproducción de la vida humana y natural, tal como se observó en el contexto donde se llevó a cabo esta investigación.

Como se expuso, la localidad de Buñuelos enfrenta una situación problemática de escasez de agua como resultado del aumento poblacional, de la sobreexplotación de los acuíferos de la zona en aras del desarrollo industrial y económico, en particular del Parque Industrial Derramadero, que inició en 2005, y del deterioro de sus ecosistemas. Su población enfrenta además la insuficiencia de condiciones materiales y sociales para una reproducción social digna.

Frente a esta marginación, en específico en lo que respecta a la escasez del agua, se observó que las mujeres y los hombres de la localidad resisten y construyen estrategias y redes para la supervivencia familiar y comunitaria, actuando de forma organizada y con un sentido de solidaridad. En este aspecto, es erróneo pensar que las funciones de cuidado que realizan las mujeres en lo do- 
méstico están desvinculadas de la vida pública. Como se mencionó, el espacio doméstico es el sitio desde el cual se tiene acceso a otros lugares productivos y relacionales (Guzmán, 2005). Esa esfera es también el espacio donde mediante el cuidado se cultiva la filiación social, expresada y materializada, familiar y comunitaria, en el sentido de solidaridad.

No obstante lo descrito, las desigualdades también resultaron expuestas; por ejemplo, el caso de las familias que tienen solvencia económica para comprar el agua o las que pueden movilizarse a otra localidad para conseguir agua potable en comparación con las que no tienen la posibilidad. Esto y las percepciones que tienen las mujeres sobre la situación actual del agua y las valoraciones socioculturales que realizan sobre sí mismas, cobran sentido en el marco del capitalismo neoliberal.

El neoliberalismo, entendido como el discurso hegemónico de un modelo civilizatorio en el que prevalecen valores básicos - progreso, conocimiento, tecnología e industria- de la sociedad liberal moderna en torno al ser humano (Lander, 2000), ha tendido a maximizar la utilidad de los recursos y a precarizar las condiciones que posibilitan la reproducción social, esto es, el abuso del capitalismo sobre la naturaleza, la anexión de la naturaleza al capital, que la expropia y utiliza de manera gratuita como fuente de insumos para la producción - pero también como muladar para absorber los residuos de ésta-, y la concibe como una esfera de valor mercantil producida por y para los seres humanos (Fraser, 2014).

Se trata de procesos de acumulación permanente que la industria moderna necesita para la producción de riqueza capitalista. En ellos se separa la producción del mercado de la reproducción de la vida humana y natural, y es donde las mujeres, junto con la naturaleza, proveen las condiciones de posibilidad y existencia del capitalismo (Carrasco, 2016; Federici, 2010; Fraser, 2014), como se muestra a continuación.

En torno a la situación actual del agua en Buñuelos, las participantes refirieron además de la escasez, la contaminación y las condiciones perniciosas en sus vidas. El hecho de que el trabajo reproductivo no esté monetizado, le ha otorgado una apariencia de naturalidad, y todo lo natural, lo gratuito, lo que forma parte de los comunes, lo expropia y lo explota el capitalismo.

Por ejemplo, en relación con los significados y las valoraciones socioculturales que las participantes atribuyen a este recurso natural, cuando dicen " $\mathrm{El}$ agua es muy necesaria para todo, hasta yo creo más que el dinero" y "El agua es como la vida [...]. Si no tienes agua, no tienes nada" se advierte el riesgo de su nulificación, debido a la valoración económica que lo convierte en mercancía con un valor y precio (Ávila, 2006). Algunas implicaciones relacionales y sociales de eso son la tendiente individualización de las familias para la provisión del agua -con el riesgo consecuente de la desarticulación de las relaciones comunales y del sentido de la cultura-, pero también el aumento de la dependencia de las mujeres del salario de los hombres para tener acceso al recurso (López y Cielo, 2018), y con ello, el riesgo latente de violencia en sus vidas debido a que se establece una jerarquía en esa relación en función del salario y el no salario, lo cual concede a los hombres el poder de disciplinarlas (Federici, 2018). 
En lo referente a las estrategias de abastecimiento familiar y comunitario del agua, se puede apreciar que aunque las mujeres y los hombres participan en la recolección a través de distintos medios, las primeras son las encargadas de su gestión y saneamiento y de solucionar el problema de las averías cuando el Comité del Agua no lo hace. Al respecto, la precariedad de las condiciones materiales recrudece las desigualdades en las posiciones desde donde se reproduce la vida (Carosio, 2010), es decir, incrementa el trabajo que realizan las mujeres para la reproducción social, lo cual, a su vez, refuerza su condición de subordinación domesticada y su explotación (Federici, 2018; Fraser, 2014; López y Cielo, 2018). Así, la relación entre capitalismo y patriarcado se manifiesta en la vida cotidiana de las mujeres de Buñuelos.

Es indiscutible la demostración de las capacidades que tienen las mujeres para la gestión y el saneamiento del agua. Pero su incidencia en el órgano de representación y participación comunitaria del recurso es limitada, debido a la vigencia de la estructura patriarcal del Comité, cuyos integrantes siempre han sido hombres. Lo anterior se fundamenta en que los derechos de uso del agua están asociados de forma directa con la tenencia de la tierra, cuya posesión siempre ha sido de los hombres (Deere y León, 2002; Gutiérrez-Villalpando et al., 2013; Martínez, 2015).

Aun cuando las mujeres reconocen la importancia que tiene el hecho de que ellas mismas representen al Comité del Agua a causa de las funciones sociales que desempeñan y debido a las formas de organización y de distribución del tiempo que tienen para cumplir con sus responsabilidades familiares y comunitarias en la gestión del recurso vital, ellas se apropian del discurso patriarcal, que tiende a justificar su exclusión cuando refieren que hay deficiencias en sus propias capacidades organizativas colectivas para participar y tomar decisiones públicas en ese espacio de poder, como se aprecia en la sentencia "Las mujeres somos más problemáticas, más chismosas". Este mecanismo de contraempoderamiento (Vázquez, 2008) mina sus esfuerzos de autonomía, las degrada de modo simbólico y las lleva a adaptar sus conductas de acuerdo con los estereotipos tradicionales de género.

Aunado a esto, desde las propias percepciones y prácticas de las mujeres, las limitaciones para tener representatividad, participar y tomar decisiones en el Comité del Agua están asociadas con la ausencia de espacios de autoorganización y gestión, que impiden la organización formal de redes, así como el desarrollo de habilidades comunicativas y de solución de problemas que faciliten la potenciación y el (auto)reconocimiento. Esto se relaciona con una estructura patriarcal que, de la mano del capitalismo, marcan tiempos distintos para las mujeres y los hombres en lo que respecta a sus funciones sociales en las esferas pública y privada.

De acuerdo con Carrasco, Borderías y Torns (2011), el propósito central del trabajo doméstico y de los cuidados en las sociedades liberales-capitalistas consiste en facilitar que los hombres adultos de la familia tengan disponibilidad laboral y libertad de tiempo y acción para participar en la vida pública sin que los cuidados ni el trabajo doméstico sean limitaciones para realizar sus actividades. Se trata de permitirles ser el homo economicus, señalan las autoras. 
Priorizar la sostenibilidad de la vida en la esfera pública daría continuidad a un conjunto de acciones en dirección a la perdurabilidad y prosperidad de nuestro entorno biológico y humano, y el reconocimiento de su imbricación. Al mismo tiempo, subvertiría las desigualdades de género que atraviesan el capitalismo, el cual presupone que las tareas naturales y gratuitas de cuidado son parte de una inmutable estructuración social de los géneros, sin tomar en cuenta que, en especial en los territorios con recursos escasos, esas tareas depositan una doble carga de responsabilidad en las mujeres que las precariza: en este caso de gestión y abasto de agua.

Se considera que la información aquí reunida es valiosa y necesaria, debido a que proviene de las mujeres y desde el lugar que ocupan en la estructura social de Buñuelos. El trabajo que asumen las entrevistadas en la gestión del agua, si se entiende el acceso al recurso como un derecho, expone las resistencias y las dificultades de gobernanza local, la discriminación por género y las desigualdades de clase que están detrás de la inseguridad en cuanto al abasto hídrico, situación que se ha reiterado en otros estudios (Gutiérrez-Villalpando et al., 2013; López y Cielo, 2018; Vázquez-García, Pérez-Olvera y Muñoz-Rodríguez, 2014).

A partir de este estudio, se entrevén líneas de investigación futuras en las cuales se registren los aportes de otros actores clave de la localidad y el análisis estructural de otras dimensiones de la calidad de vida en Buñuelos que integre también distintas miradas y aproximaciones teóricas y metodológicas, para ampliar la perspectiva de los resultados aquí presentados.

\section{Agradecimientos}

Las autoras agradecen el apoyo financiero del Consejo Nacional de Ciencia y Tecnología de Coahuila, otorgado en el marco de la Convocatoria FONCYT 2019 C13.

\section{Referencias}

Aboites, L., Birrichaga, D., y Garay, J. (2010). El manejo de las aguas mexicanas en el siglo XX. En B. Jiménez, M. L. Torregrosa y Armentia y L. Aboites (eds.), El agua en México: cauces y encauces (pp. 21-49). México: Academia Mexicana de Ciencias y Comisión Nacional de Agua.

Aguirre, V. L., Tobón de Garza, G., y Mendoza, A. R. (2018a). El agua, factor limitante para el futuro desarrollo regional sostenible del sureste de Coahuila, y de la Zona Metropolitana de Saltillo. En J. E. Isaac y L. R. Rózga (coords.), Empresas, actores sociales e instituciones en la organización productiva del territorio y la innovación para el desarrollo local, Vol. III (pp. 19-34). México: Universidad Nacional Autónoma de México y Asociación Mexicana de Ciencias para el Desarrollo Regional, A. C. 
Aguirre, V. L., Tobón de Garza, G., y Mendoza, A. R. (2018b). Dinámica de las regiones de Coahuila: entre las fuerzas de la cuarta revolución industrial, el asalto a sus recursos naturales y las luchas por el espacio. En C. G. Hoyos, O. Serrano, E. Serena y C. M. Mora (coords.), Ciudad, género, cultura y educación en las regiones (pp. 148-175). México: Universidad Nacional Autónoma de México y Asociación Mexicana de Ciencias para el Desarrollo Regional, A. C.

Alberti, P., Zavala, M., Salcido, B., y Real, N. (2014). Género, economía del cuidado y pago del trabajo doméstico rural en Jilotepec, Estado de México. Agricultura, Sociedad y Desarrollo, 11(30), 379-400. Recuperado de http: / /www. scielo.org.mx/scielo.php?script=sci_arttext\&pid=S1870-54722014000300007

Ávila, G. P. (2006). El valor social y cultural del agua. En D. Soares, V. Vázquez, A. Serrano y R. de la Rosa (eds.), Gestión y cultura del agua, tomo II. (pp. 233-248). México: Instituto Mexicano de Tecnología del Agua y Colegio de Postgraduados en Ciencias Agrícolas.

Bartra, A. (2013). Crisis civilizatoria. En R. Ornelas (coord.), Crisis civilizatoria y superación del capitalismo (pp. 25-71). México: Universidad Nacional Autónoma de México, Instituto de Investigaciones Económicas.

Bartra, A. (20 de junio de 2020). El virus, la sociedad, el Estado. La Jornada del Campo, 153, 2.

Cáceres, P. (2008). Análisis cualitativo de contenido: una alternativa metodológica alcanzable. Psicoperspectivas. Individuo y Sociedad, 2(1), 53-82. Recuperado de http://psicoperspectivas.cl/index.php/ psicoperspectivas/ article/viewFile/3/3

Carosio, A. (2010). Frente a la crisis económica y civilizatoria: un nuevo contrato socialista y feminista. En A. Girón (coord.), Crisis económica. Una perspectiva feminista desde América Latina (pp. 51-73). Caracas: Universidad Nacional Autónoma de México, Consejo Latinoamericano de Ciencias Sociales y Universidad Central de Venezuela.

Carrasco, B. C. (2011). La economía del cuidado: planteamiento actual y desafíos pendientes. Revista de Economía Crítica 11, 205-225. Recuperado de http:/ / www.revistaeconomiacritica.org/sites/default/files/revistas/n11/ REC11_9_intervenciones_CristinaCarrasco.pdf

Carrasco, B. C. (2014). La economía feminista: ruptura teórica y propuesta política. En B. C. Carrasco (ed.), Con voz propia: la economía feminista como apuesta teórica y política (pp. 25-47). Madrid: Kadmos.

Carrasco, B. C. (2016). Sostenibilidad de la vida y ceguera patriarcal. Una reflexión necesaria. Atlánticas, Revista Internacional de Estudios Feministas, 1(1), 34-57. https://doi.org/10.17979/arief.2016.1.1.1435

Carrasco, C., Borderías, C., y Torns, T. (2011) Introducción. El trabajo de cuidados: antecedentes históricos y debates actuales. En C. Carrasco, C. Borderías y T. Torns (eds.), El trabajo de cuidados. Historia, teoría y políticas (pp. 13-96). Madrid: Catarata.

Cleaver, F. (1998). Choice, complexity, and change: gendered livelihoods and the management of water. Agriculture and Human Values, 15, 293-299. doi: https://doi.org/10.1023/A:1007511816437 
Consejo Nacional de Población (CONAPO). (2012). Estimaciones de la CONAPO con base en el Censo de Población y Vivienda 2010. Recuperado de https: / / www.inegi.org.mx/programas/ccpv/2010/

Deere, D., y León, M. (2002). Género, propiedad y empoderamiento. Tierra, Estado y Mercado en América Latina. México: UNAM.

Esparza, M. (2014). La sequía y escasez de agua en México. Situación actual y perspectivas futuras. Secuencia, 89, 195-219. Recuperado de http://www. scielo.org.mx/scielo.php?script=sci_arttext\&pid=S0186-03482014000200008

Federici, S. (2010). Calibán y la bruja. Mujeres, cuerpo y acumulación originaria. Madrid: Traficantes de Sueños.

Federici, S. (2018). El patriarcado del salario. Críticas feministas al marxismo. Madrid: Tinta Limón. Traficantes de Sueños.

Federici, S. (2020). Reencantar el mundo. El feminismo y la política de los comunes. Madrid: Traficantes de Sueños.

Flick, U. (2004). Introducción a la investigación cualitativa. Madrid: Morata.

Fraser, N. (2014). Tras la morada oculta de Marx. Por una concepción ampliada del capitalismo. New Left Review, 86, 59-78.

Fundación Villa Patos, A. C., e Instituto Mexicano para el Desarrollo Comunitario, A. C. (2018). Informe Derramadero. La Ciudad Modelo y el Agua en Saltillo, Coahuila. Recuperado de https://agua.org.mx/biblioteca/informe-derramadero-la-ciudad-modelo-y-el-agua-en-saltillo-coahuila/

García, A., y Vázquez, V. (2017). Derecho humano al agua y desigualdad social en San Jerónimo Teócatl, Oaxaca. Cuicuilco, Revista de Ciencias Antropológicas, 24(68),157-176. Recuperado de http://www.scielo.org.mx/scielo. php?script=sci_arttext\&pid=S2448-84882017000100157\&lng=es\&nrm=iso\&tl ng=es

Girón, A. (2010). Circuitos de la crisis: resquebrajamiento del modelo económico y perspectiva feminista. En A. Girón (coord.), Crisis económica. Una perspectiva feminista desde América Latina (pp. 29-49). Caracas: Universidad Nacional Autónoma de México, Consejo Latinoamericano de Ciencias Sociales y Universidad Central de Venezuela.

Gleick, H. P., y Heberger, M. (2014). Water and conflict. events, trends, and analysis (2011-2012). En H. P. Gleick, The World's Water, Vol. 8 (pp. 159171). Washington, D. C.: Island Press.

Gobierno del Estado Coahuila de Zaragoza. (2018). Plan Estatal de Desarrollo 2017-2023. Recuperado de https://coahuila.gob.mx/archivos/pdf/Publicaciones/PED2017-2023/Plan_Estatal_Desarrollo_baja.pdf

González, A. S., (2017). Resumen ejecutivo. Contexto físico. En La biodiversidad en Coahuila. Estudio de Estado, Vol. I. (pp. 31-32). Comisión Nacional para el Conocimiento y Uso de la Biodiversidad y Gobierno del Estado de Coahuila de Zaragoza, México. Recuperado de https://www.cbd.int/doc/ nbsap/study/mx-study-coahuila-p2-es.pdf

Gutiérrez-Villalpando, V., Nazar-Beutelspacher, D. A., Zapata-Martelo, E., Contreras-Utrera, J., y Salvatierra-Izaba, B. (2013). Mujeres y organización social en la gestión del agua para consumo humano y uso doméstico en Berrio- 
zábal, Chiapas. LiminaR, 11(2), 100-113. Recuperado de http://www.scielo. org.mx/pdf/liminar/v11n2/v11n2a7.pdf

Guzmán, G. E. (2005). Resistencia, permanencia y cambio. Estrategias campesinas de vida en el poniente de Morelos. México: Universidad Autónoma del Estado de México y Plaza y Valdés.

Harding, S. (2010). ¿Una filosofía de la ciencia socialmente relevante? Argumentos en torno a la controversia sobre el punto de vista feminista. En G. N. Blazquez, P. F. Flores y E. M. Ríos (coords.), Investigación feminista, metodología y representaciones sociales (pp. 39-65). México: Universidad Nacional Autónoma de México, Centro de Investigaciones Interdisciplinarias en Ciencias Sociales y Humanidades, Centro Regional de Investigaciones Multidisciplinarias, Facultad de Psicología.

Haraway, D. (1995). Ciencia, cyborgs y mujeres, la reinvención de la naturaleza. Madrid: Ediciones Cátedra, Universitat de Valencia e Instituto de la Mujer.

Instituto Nacional de Estadística y Geografía (INEGI). (2016). Sistema de Cuentas Nacionales de México. Participación por actividad económica en valores corrientes.

Instituto Nacional de Estadística y Geografía (INEGI). (2020). Censo de Población y Vivienda. Recuperado de http://www.cuentame.inegi.org.mx/monografias $/$ informacion / coah / poblacion / distribucion. aspx?tema=me\&e=05

Kvale, S. (2007). Doing Interviews. Thousand Oaks, CA: Sage Publications.

Lander, E. (2000). Ciencias sociales: saberes coloniales y eurocéntrico. En E. Lander (comp.), La colonialidad del saber: eurocentrismo y ciencias sociales. Perspectivas Latinoamericanas (pp. 4-23). Buenos Aires: CLACSO.

López, C., E., y Cielo, C. (2018). El agua, el cuidado y lo comunitario en la Amazonía boliviana y ecuatoriana. En S. Vega, B. Martínez y C. Paredes (eds.), Cuidados, comunidad, común (pp. 53-73). Madrid: Traficantes de Sueños.

Martínez, B. (2015). Género, ambiente, mujeres rurales y recursos naturales. En E. Zapata-Martelo y M. Ayala-Carrillo (coords.), Contribuciones de los estudios de género al desarrollo rural (pp. 217-318). México: Colegio de Postgraduados.

Martínez, L. (2007). La observación y el diario de campo en la definición de un tema de investigación. Revista Perfiles Libertadores, 4(80), 73-80.

Martínez, M. (2004). Los grupos focales de discusión como método de investigación. Heteropía, 10(26), 59-72.

Moreno, J. L., Marañón, B., y López, D. (2010). Los acuíferos sobreexplotados: origen, crisis y gestión social. En C. Jiménez, M. Torregrosa y Armentia y L. Aboites (eds.), El agua en México: cauces y encauces (pp. 79-115). México: Academia Mexicana de Ciencias y Comisión Nacional del Agua.

López, V., y Rojas, L. V. (2017). Rezagos en el nivel de autonomía de las mujeres rurales mexicanas en la primera década del siglo XXI. Estudios Demográficos y Urbanos, 32(2), 315-354.

Okuda, M. , y Gómez-Restrepo, C. (2005). Métodos en investigación cualitativa: triangulación. Revista Colombiana de Psiquiatría, 34(1), 118-124. Recuperado de http://www.redalyc.org/pdf/806/80628403009.pdf 
Ortega, R. G., y Portillo, A. (2015). El agua: bien común o mercancía. Paraguay: BASE-IS.

Patton, M. (1990). Qualitative Evaluation and Research Methods. Beverly Hills: Sage.

Rowlands, J. (1997). Empoderamiento y mujeres rurales en Honduras: un modelo para el desarrollo. En M. León (comp.), Poder y empoderamiento de las mujeres (pp. 213-245). Santa Fé de Bogotá: Tercer Mundo.

Sánchez, L. F., y Fernández, S. B. (2018). La participación comunitaria y social de las mujeres en la gestión del agua como bien del procomún en Honduras. En P. J. Martínez y C. J. Moreno (coords.), Comprender el presente, imaginar el futuro: nuevas y viejas brechas sociales (pp. 815-834). Roma y Mesina: CORISCO Edizione.

Secretaría de Desarrollo Social (SEDESOL). (2013). Catálogo de Localidades del Sistema de Apoyo para la Planeación del Programa de Desarrollo de Zonas Prioritarias. Recuperado de http://www.microrregiones.gob.mx/catloc/ LocdeMun.aspx?buscar=1\&tipo=nombre\&campo=loc\&valor=Buñuelos

Secretaría de Medio Ambiente y Recursos Naturales (SEMARNAT) y Comisión Nacional del Agua (CONAGUA). (2017). Estadísticas del Agua en México. Edición 2017. Recuperado de http://sina.conagua.gob.mx/publicaciones/ EAM_2017.pdf

Segato, R. (2018). Contra-pedagogías de la crueldad. Buenos Aires: Prometeo libros.

Soares, D. (2007). Acceso, abasto y control del agua en una comunidad indígena chamula en Chiapas. Un análisis a través de la perspectiva de género, ambiente y desarrollo. región y sociedad, 19(38), 25-50. Recuperado de http: / / www. scielo.org.mx/scielo.php?script=sci_arttext\&pid=S1870-39252007000100002

Organización de las Naciones Unidas para la Educación, la Ciencia y la Cultura (UNESCO). (2018). Informe mundial de las Naciones Unidas sobre el Desarrollo de los Recursos Hídricos 2018: soluciones basadas en la naturaleza para la gestión del agua, cifras y datos. Recuperado de https://unesdoc.unesco. org/ark: / 48223/pf0000261494

Picchio, A. (2009). Condiciones de vida: perspectivas, análisis económico y políticas públicas. Revista de Economía Crítica, 7, 27-54. Recuperado de http://revistaeconomiacritica.org/sites/default/files/revistas/n7/2_condiciones_de_vida.pdf

Ruiz, L. (2012). Relaciones de género y mercados de derecho de aguas y tierra en Chiapas. región y sociedad, 24(53), 55-89. doi: https://doi.org/10.22198/ rys.2012.53.a162

Valdivieso, M. (2010). Mujeres, desarrollo y crisis. En A. Girón (coord.), Crisis económica. Una perspectiva feminista desde América Latina (pp. 75-93). Caracas: Universidad Nacional Autónoma de México, Consejo Latinoamericano de Ciencias Sociales y Universidad Central de Venezuela.

Vázquez, V. (2008). El chisme y la violencia de género. En R. Castro e I. Casique (eds.), Estudios sobre cultura, género y violencia contra las mujeres (pp. 139-172). México: Universidad Nacional Autónoma de México. 
Vázquez, G. V., Cárcamo, T. N., y Buendía, R. A. (2007). Desarrollo sustentable y perspectiva de género. Algunos aportes conceptuales y de política pública. Géneros. Revista de Investigación y Divulgación sobre los Estudios de Género, 2(14), 38-48. Recuperado de http:/ / bvirtual.ucol.mx/descargables/872 desarrollo_sustentable.pdf

Vázquez-García, V., Pérez-Olvera, M. A., y Muñoz-Rodríguez, C. (2014). Desarrollo, género y el derecho humano al agua. Un estudio comparativo en Hidalgo, México. Agricultura, Sociedad y Desarrollo, 11(3), 295-314. Recuperado de http://www.scielo.org.mx/ scielo.php? script= sci_arttext\&pi$\mathrm{d}=\mathrm{S} 1870-54722014000300003$

Vázquez-García, V., y Sosa-Capistrán, M. D. (2017). Sin agua no vivo: género y derecho humano al agua en el municipio de la Antigua, Veracruz. Agricultura, Sociedad y Desarrollo, 14(3), 405-425. Recuperado de http://www. scielo.org.mx/scielo.php?script=sci_arttext\&pid=S1870-54722017000300405

Vázquez-Luna, D., Mortera, P. D., Rodríguez-Orozco, N., Martínez, M. M., y Velázquez, S. M. (2013). Organización comunitaria de mujeres: del empoderamiento al éxito del desarrollo rural sustentable. La Ventana, 4(37), 262-288. Recuperado de http://www.scielo.org.mx/pdf/laven/v4n37/v4n37a11.pdf

Villarreal Reyna, F. A. (2013). Evaluación del potencial hídrico y su impacto en el desarrollo de Saltillo, Coahuila (tesis de doctorado). Recuperado de http://eprints.uanl.mx/3745/ 